\title{
Evidence for strong lattice effects as revealed from huge unconventional oxygen isotope effects on the pseudogap temperature in $\mathrm{La}_{2-x} \mathrm{Sr}_{x} \mathrm{CuO}_{4}$
}

\author{
M. Bendele,,${ }^{1,2,{ }^{*}}$ F. von Rohr, ${ }^{1}$ Z. Guguchia, ${ }^{3}$ E. Pomjakushina,${ }^{4}$ K. Conder ${ }^{4}$ A. Bianconi,${ }^{2,5}$ A. Simon, ${ }^{6}$ \\ A. Bussmann-Holder, ${ }^{6}$ and H. Keller ${ }^{1}$ \\ ${ }^{1}$ Physik-Institut der Universität Zürich, Winterthurerstrasse 190, CH-8057 Zürich, Switzerland \\ ${ }^{2}$ Rome International Center for Materials Science Superstripes (RICMASS), Via dei Sabelli 119A, I-00185 Rome, Italy \\ ${ }^{3}$ Laboratory for Muon Spin Spectroscopy, Paul Scherrer Institute, CH-5232 Villigen PSI, Switzerland \\ ${ }^{4}$ Laboratory for Scientific Developments and Novel Materials, Paul Scherrer Institute, CH-5232 Villigen PSI, Switzerland \\ ${ }^{5}$ Department of Physics of Solid State and Nanosystems, National Research Nuclear University, Kashirskoye shosse 31, \\ Moscow 115409, Russia \\ ${ }^{6}$ Max-Planck Institute for Solid State Research, Heisenbergstrasse 1, D-70569 Stuttgart, Germany \\ (Received 9 May 2016; revised manuscript received 13 December 2016; published 19 January 2017)
}

\begin{abstract}
The oxygen isotope $\left({ }^{16} \mathrm{O} /{ }^{18} \mathrm{O}\right)$ effect (OIE) on the pseudogap (charge-stripe ordering) temperature $T^{*}$ is investigated for the cuprate superconductor $\mathrm{La}_{2-x} \mathrm{Sr}_{x} \mathrm{CuO}_{4}$ as a function of doping $x$ by means of $\mathrm{x}$-ray absorption near edge structure studies. A strong $x$ dependent and sign reversed OIE on $T^{*}$ is observed. The OIE exponent $\alpha_{T^{*}}$ systematically decreases from $\alpha_{T^{*}}=-0.6(1.3)$ for $x=0.15$ to $\alpha_{T^{*}}=-4.4(1.1)$ for $x=0.06$, corresponding to increasing $T^{*}$ and decreasing superconducting transition temperature $T_{c}$. Both $T^{*}\left({ }^{16} \mathrm{O}\right)$ and $T^{*}\left({ }^{18} \mathrm{O}\right)$ exhibit a linear doping dependence with different slopes and critical end points [where $T^{*}\left({ }^{16} \mathrm{O}\right)$ and $T^{*}\left({ }^{18} \mathrm{O}\right)$ fall to zero] at $x_{c}\left({ }^{16} \mathrm{O}\right)=0.201(4)$ and $x_{c}\left({ }^{18} \mathrm{O}\right)=0.182(3)$, indicating a large positive OIE of $x_{c}$ with an exponent of $\alpha_{x_{c}}=0.84(22)$. The remarkably large and strongly doping dependent OIE on $T^{*}$ signals a substantial involvement of the lattice in the formation of the pseudogap, consistent with a polaronic approach to cuprate superconductivity and the vibronic character of its ground state.
\end{abstract}

DOI: 10.1103/PhysRevB.95.014514

\section{INTRODUCTION}

Even 30 years after its discovery, the origin of hightemperature superconductivity in cuprate superconductors (HTSs) is still under debate [1]. A seemingly majority of researchers advocates for a purely electronic mechanism, and consequently ignores the importance of lattice effects [2]. However, in conventional superconductors the role of the lattice as a pairing glue for Cooper pairs has been identified by the observation of an isotope effect on the superconducting transition temperature $T_{c}$ [3]. Interestingly, in cuprate superconductors, analogous but apparently unconventional and doping dependent isotope effects have been observed on several characteristic quantities, e.g., the superconducting transition temperature $T_{c}$, the antiferromagnetic transition temperature $T_{N}$, the spin-glass temperature $T_{g}$, the spin-stripe ordering temperature $T_{\mathrm{so}}$, and the magnetic penetration depth $\lambda$ [4-11], which all are in support of the vibronic character of its ground state. So far, only a few studies of the isotope effect on the pseudogap temperature $T^{*}$ have been reported [12-19].

The pseudogap temperature $T^{*}$ plays a central role in the phase diagram of HTSs and is considered to be a key ingredient for the understanding of the physics of cuprates [20]. From an experimental point of view, $T^{*}$ itself is a rather ill-defined temperature scale with varying values depending on the experimental tool and the involved time scale [12-15]. The nature of $T^{*}$ in cuprates is debated and attributed to controversial origins [21]. These are discussed as precursors to the paired state, spin density formation, charge density

*markus.bendele@gmail.com formation, onset of stripe formation, and other exotic scenarios [21]. Here, we define the pseudogap $T^{*}$ as the temperature where lattice effects are apparent in terms of deviations of the local structure from the average one (this temperature is also denoted as charge-stripe ordering temperature in the literature [14]). The relevance of lattice/polaron effects for the appearance of the pseudogap phase can be tested by oxygen isotope $\left({ }^{16} \mathrm{O} /{ }^{18} \mathrm{O}\right)$ effect (OIE) studies. So far, only a limited number of such experiments have been performed [12-19]. First, ${ }^{89} \mathrm{Y}$ nuclear magnetic resonance (NMR) measurements on slightly underdoped $\mathrm{YBa}_{2} \mathrm{Cu}_{4} \mathrm{O}_{8}$ show no appreciable OIE on $T^{*}$ [12], whereas ${ }^{63} \mathrm{Cu}$ nuclear quadrupole resonance (NQR) spin-lattice relaxation experiments on the same system revealed a small but positive OIE on $T^{*}$ [13]. However, a large and sign reversed OIE has been reported from $\mathrm{Cu} K$-edge $\mathrm{X}$-ray absorption near edge structure (XANES) studies in underdoped $\mathrm{La}_{1.94} \mathrm{Sr}_{0.06} \mathrm{CuO}_{4}$ [14], and from neutron crystal field spectroscopy (NCFS) studies in slightly underdoped $\mathrm{HoBa}_{2} \mathrm{Cu}_{4} \mathrm{O}_{8}$ [15], optimum doped $\mathrm{La}_{1.81} \mathrm{Ho}_{0.04} \mathrm{Sr}_{0.15} \mathrm{CuO}_{4}$ [18], and doped $\mathrm{La}_{1.96-x} \mathrm{Ho}_{0.04} \mathrm{Sr}_{x} \mathrm{CuO}_{4}(0.11 \leqslant x \leqslant 0.25)$ [19]. Moreover, NCFS studies of the ${ }^{63} \mathrm{Cu} /{ }^{65} \mathrm{Cu}$ isotope effect evidenced a large negative isotope shift of $T^{*}$ in $\mathrm{HoBa}_{2} \mathrm{Cu}_{4} \mathrm{O}_{8}$ [17], whereas no copper isotope shift of $T^{*}$ was observed for optimum doped $\mathrm{La}_{1.81} \mathrm{Ho}_{0.04} \mathrm{Sr}_{0.15} \mathrm{CuO}_{4}$ [18].

Here, we present a systematic study of the OIE on $T^{*}$ over a broad doping range of $\mathrm{La}_{2-x} \mathrm{Sr}_{x} \mathrm{CuO}_{4}(0.06 \leqslant x \leqslant 0.15)$ by means of $\mathrm{Cu} K$-edge XANES studies [22].

\section{EXPERIMENTAL DETAILS}

Four different $\mathrm{La}_{2-x} \mathrm{Sr}_{x} \mathrm{CuO}_{4}$ samples with concentrations $x=0.06,0.09,0.12$, and 0.15 were prepared by a conventional 


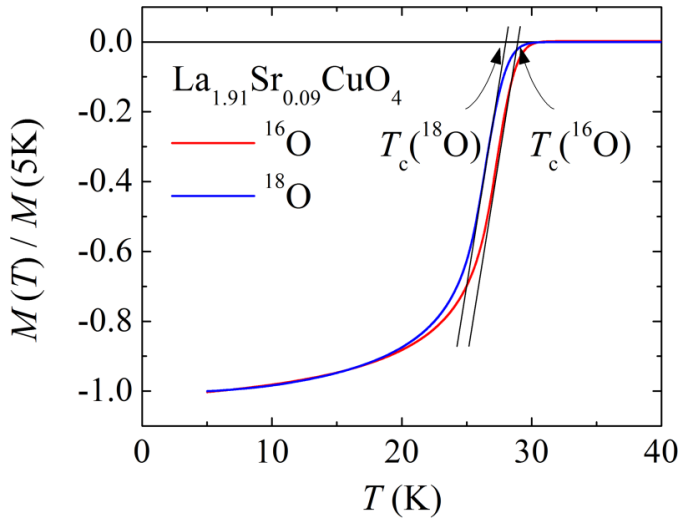

FIG. 1. Temperature dependence of the zero-field-cooled normalized magnetization $M(T) / M(5 \mathrm{~K})$ of the ${ }^{16} \mathrm{O} /{ }^{18} \mathrm{O}$ exchanged samples of $\mathrm{La}_{1.91} \mathrm{Sr}_{0.09} \mathrm{CuO}_{4}$ in $\mu_{0} H=0.5 \mathrm{mT}$. The superconducting transition temperature was determined by the intersection of the linearly extrapolated magnetic moment and the zero line.

solid state reaction using dried $\mathrm{La}_{2} \mathrm{O}_{3}(99.99 \%), \mathrm{SrCO}_{3}$ (99.999\%), and $\mathrm{CuO}$ (99.999\%). The powders were mixed, ground thoroughly, pressed into pellets. and fired in air at $1000{ }^{\circ} \mathrm{C}$ for $\sim 100 \mathrm{~h}$ with three intermediate grindings. The phase purity of the samples was checked with a conventional $\mathrm{x}$-ray diffractometer. The final ${ }^{18} \mathrm{O}$ and ${ }^{16} \mathrm{O}$ samples were obtained in parallel via oxygen isotope exchange in closed quartz glass tubes under a controlled gas pressure slightly above 1 bar at $800^{\circ} \mathrm{C}$ during $72 \mathrm{~h}$ with subsequent cooling. The oxygen isotope enrichment was determined from the weight changes and was about $90 \%$ of ${ }^{18} \mathrm{O}$ for all samples.

The samples were characterized by measuring their superconducting properties using a superconducting quantum interference device (SQUID) magnetometer (Quantum Design MPMS-XL). The temperature dependencies of the zerofield-cooled normalized magnetization $M(T) / M(5 \mathrm{~K})$ of the ${ }^{16} \mathrm{O} /{ }^{18} \mathrm{O}$ exchanged samples of $\mathrm{La}_{1.91} \mathrm{Sr}_{0.09} \mathrm{CuO}_{4}$ for $\mu_{0} H=$ $0.5 \mathrm{mT}$ are shown in Fig. 1, with $T_{c}$ being identified from the zero of the linearly extrapolated magnetic moment. From the values of $T_{c}\left({ }^{16} \mathrm{O}\right)$ and $T_{c}\left({ }^{18} \mathrm{O}\right)$ the OIE exponent $\alpha_{T_{c}}$ is derived according to $\alpha_{T_{c}}=-d \ln T_{c} / d \ln M_{\mathrm{O}}$, where $M_{\mathrm{O}}$ is the mass of the oxygen isotope. The results of the OIE on $T_{c}$ for $\mathrm{La}_{2-x} \mathrm{Sr}_{x} \mathrm{CuO}_{4}(0.06 \leqslant x \leqslant 0.15)$ will be discussed in more detail below, in connection with the OIE on $T^{*}$ observed in this work.

In order to ascertain $T^{*}, \mathrm{Cu} K$-edge XANES measurements were performed on powder samples at the Diamond Light Source on the B18 beamline [23]. The storage ring was operating in a 10-min top-up mode for a ring current of $300 \mathrm{~mA}$ and an energy of $3 \mathrm{GeV}$. The radiation was monochromated with a $\mathrm{Si}$ (311) double crystal monochromator calibrated using the $K$ edge of a copper foil, taking the first inflection point as $8979.0 \mathrm{eV}$. Harmonic rejection was achieved through the use of two platinum-coated mirrors operating at an incidence angle of $7.5 \mathrm{mrad}$. The incident $\left(I_{0}\right)$, the transmitted $\left(I_{t}\right)$, and the reference $\left(I_{\text {ref }}\right)$ beam intensities were measured using two $30 \mathrm{~cm}$ ion chambers and a helium/argon gas mixture (total ion chamber pressure of $1.1 \mathrm{bar}$ ) to absorb $12 \%, 60 \%$, and $60 \%$ of the beam in $I_{0}, I_{t}$ and $I_{\text {ref }}$, respectively [23]. The samples
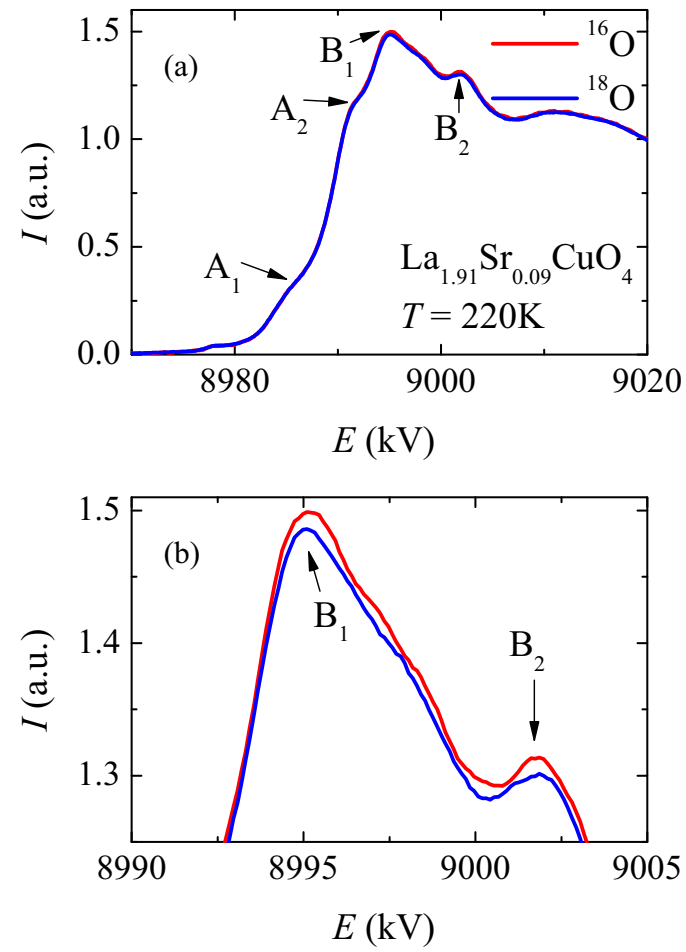

FIG. 2. (a) Normalized $\mathrm{Cu} K$-edge XANES spectra showing the energy evolution of the normalized intensity $I$ for both isotopes ${ }^{16} \mathrm{O}$ and ${ }^{18} \mathrm{O}$ of $\mathrm{La}_{1.91} \mathrm{Sr}_{0.09} \mathrm{CuO}_{4}$ at $T=220 \mathrm{~K}$. (b) Extended region of the XANES spectrum depicted in (a) showing a slight change in the shape due to oxygen isotope substitution $\left({ }^{18} \mathrm{O} /{ }^{18} \mathrm{O}\right)$.

were positioned between $I_{0}$ and $I_{t}$ and the $\mathrm{Cu}$ foil reference is between $I_{t}$ and $I_{\text {ref }}$.

Five absorption spectra were collected at the same temperature in an Oxford Instruments OptistatPT cryostat and their mean value taken in order to increase the signal-to-noise ratio. Figure 2 shows the normalized $\mathrm{Cu} K$-edge XANES spectra for the ${ }^{16} \mathrm{O}$ and ${ }^{18} \mathrm{O} \mathrm{La}_{1.91} \mathrm{Sr}_{0.09} \mathrm{CuO}_{4}$ samples at $T=220 \mathrm{~K}$ as a representative for the entire series. The spectra show the typical absorption features of the cuprates with a square planar geometry where the main features are denoted as $A_{1}, A_{2}, B_{1}$, and $B_{2}$ with peak intensities $a_{1}, a_{2}, b_{1}$, and $b_{2}$. The peak $A_{1}$ is essentially caused by the multiple scatterings of the ejected photoelectrons from the $\mathrm{La} / \mathrm{Sr}$ atoms, while peak $A_{2}$ is due to the apical oxygen. The features above the threshold from 8 to $40 \mathrm{eV}$ have been identified as multiple scattering resonances within a $0.5 \mathrm{~nm}$ cluster of neighbor atoms surrounding the absorbing atom [22], i.e., $B_{1}$ probes the scattering from the oxygen atoms in the square $\mathrm{CuO}_{2}$ planes. The decrease of the $b_{1}$ and $b_{2}$ resonance intensities, together with the increase of both $A_{1}$ and $A_{2}$ multiple scattering photoelectron resonance intensities $a_{1}$ and $a_{2}$, indicate deviations from the $\mathrm{CuO}_{2}$ square plane geometry for anisotropic atomic displacements in the $\mathrm{CuO}_{4}$ plaquette [22], which has been used to probe the onset of periodic local lattice distortions associated with polaron formation.

The effect of the oxygen isotope substitution on the XANES spectra on the distribution of local $\mathrm{Cu}$-site conformations is hardly seen directly in the raw XANES spectra shown in Fig. 2, but can be extracted from the slightly different 

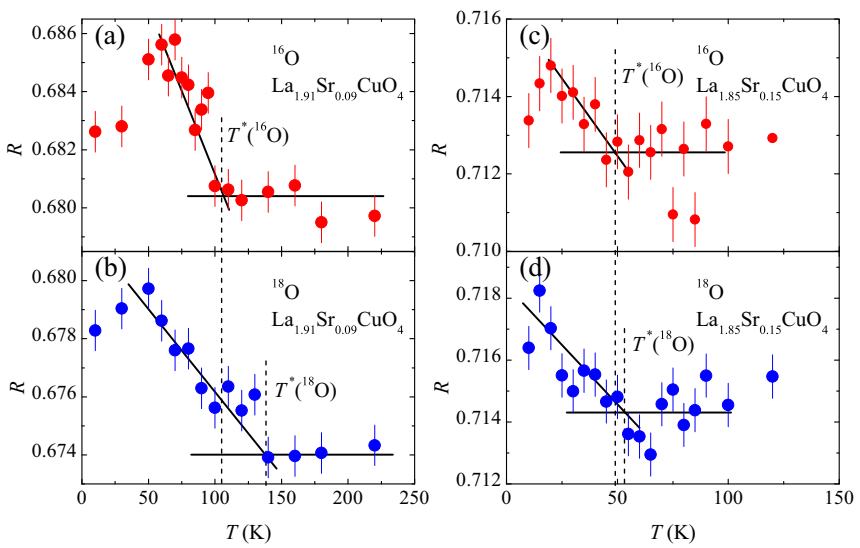

FIG. 3. The temperature dependence of the XANES peak intensity ratio $R$ of oxygen isotope substituted $\mathrm{La}_{2-x} \mathrm{Sr}_{x} \mathrm{CuO}_{4} \cdot x=0.09$ : (a) ${ }^{16} \mathrm{O}$ and (b) ${ }^{18} \mathrm{O} ; x=0.15$ : (c) ${ }^{16} \mathrm{O}$ and (d) ${ }^{18} \mathrm{O}$. The solid straight lines are a guide to the eye. The corresponding charge-stripe ordering temperatures $T^{*}$ are marked by the vertical dashed lines. The huge OIE on $T^{*}$ is evident.

XANES peak intensities $a_{1}$ and $b_{1}$ of the ${ }^{16} \mathrm{O}$ and ${ }^{18} \mathrm{O}$ samples [14]. Deviations from the square plane geometry of the $\mathrm{CuO}_{4}$ coordination plaquette can be quantified by the XANES peak ratio [14]

$$
R=\left(b_{1}-a_{1}\right) /\left(b_{1}+a_{1}\right) .
$$

In Fig. 3 the XANES intensity ratio $R$ is plotted for ${ }^{16} \mathrm{O}$ and ${ }^{18} \mathrm{O}$ samples of $\mathrm{La}_{2-x} \mathrm{Sr}_{x} \mathrm{CuO}_{4}(x=0.9$ and $x=0.15)$ as a function of temperature. From the temperature dependence of $R$, the pseudogap temperature $T^{*}$ is determined by the interception of the horizontal line above $T^{*}$ and to the linear slope below $T^{*}$ as shown in Fig. 3. For all doping levels $x$, a clear increase in $R$ below $T^{*}$ is observed, which is typical for a charge density wave and consistent with recent resonant $\mathrm{x}$-ray scattering studies, where charge ordering and the pseudogap formation were intensively studied [24]. Below $T_{c}$ the quantity $R$ decreases sharply, caused by the decrease of charge carriers due to the formation of the superconducting condensate.

\section{RESULTS}

The values of the pseudogap temperature $T^{*}$ as well as the corresponding values of $T_{c}$ as measured for all ${ }^{16} \mathrm{O}$ and ${ }^{18} \mathrm{O}$ samples of $\mathrm{La}_{2-x} \mathrm{Sr}_{x} \mathrm{CuO}_{4}(0.06 \leqslant x \leqslant 0.15)$ are summarized in Fig. 4(a) and Table I. $T_{c}(x)$ shows the well-known domelike behavior with a small but clearly visible OIE on $T_{c}$. $T^{*}$ decreases with increasing $x$ as is well known from the literature. The two isotopes show the same trends with $x$, however, $T^{*}\left({ }^{18} \mathrm{O}\right)$ is pronouncedly raised as compared to $T^{*}\left({ }^{16} \mathrm{O}\right)$ with the largest OIE shift of $\Delta T^{*} \simeq 61 \mathrm{~K}$ for $x=0.06$. With increasing $x$, the OIE on $T^{*}$ decreases systematically. For comparison, the values for $T^{*}\left({ }^{16} \mathrm{O}\right)$ and $T^{*}\left({ }^{18} \mathrm{O}\right)$ obtained from previous XANES experiments for $\mathrm{La}_{1.94} \mathrm{Sr}_{0.06} \mathrm{CuO}_{4}$ [14] and from previous NCFS experiments for $\mathrm{La}_{1.96-x} \mathrm{Ho}_{0.04} \mathrm{Sr}_{x} \mathrm{CuO}_{4}$ $(x=0.15[18] ; x=0.11,0.15$ [19] $)$ are also displayed in Fig. 4(a). These previous values for $T^{*}\left({ }^{16} \mathrm{O}\right)$ and $T^{*}\left({ }^{18} \mathrm{O}\right)$ are, within experimental error, in good agreement with the present results, demonstrating that the two different experimental

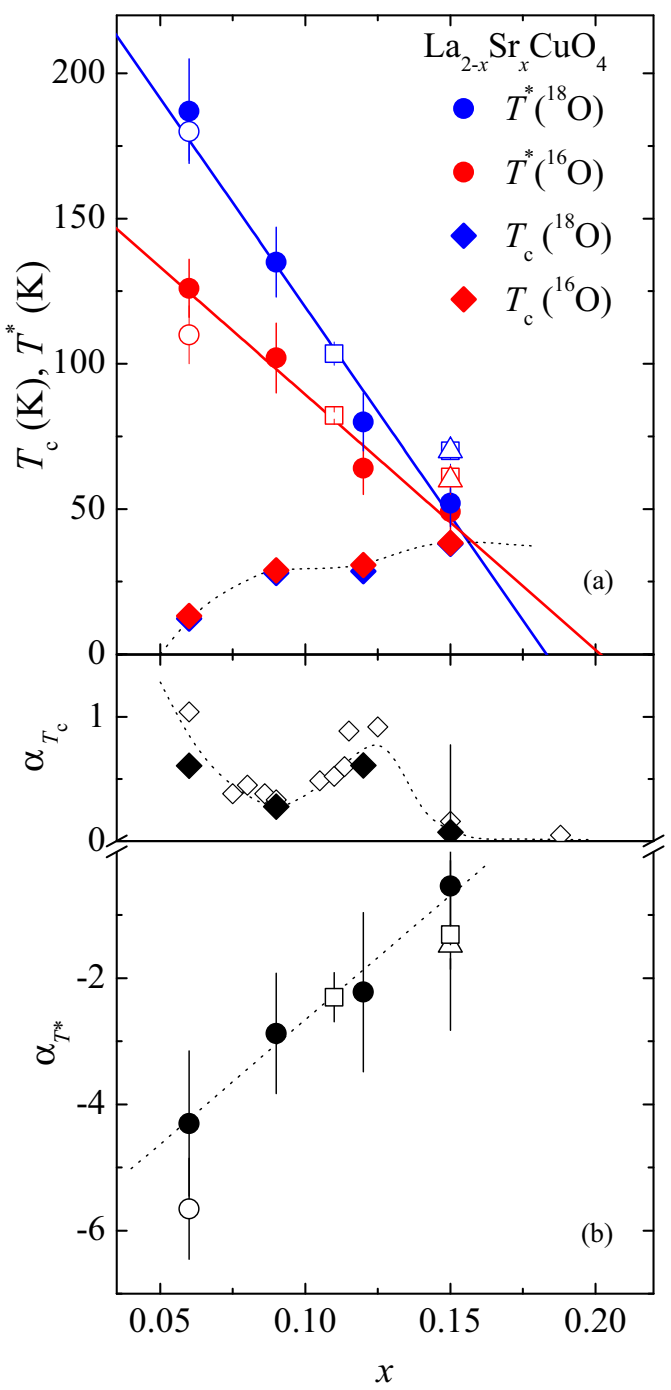

FIG. 4. (a) The superconducting transition temperature $T_{c}$ and the pseudogap temperature $T^{*}$ of $\mathrm{La}_{2-x} \mathrm{Sr}_{x} \mathrm{CuO}_{4}$ as a function of doping $x$ for ${ }^{16} \mathrm{O}$ (red symbols) and ${ }^{18} \mathrm{O}$ (blue symbols). With increasing doping $x$, a continuous decrease of $T^{*}$ is observed for both isotopes. The solid lines are obtained from Eq. (2). The dashed line is a guide to the eye. For comparison, the values for $T^{*}\left({ }^{16} \mathrm{O}\right)$ and $T^{*}\left({ }^{18} \mathrm{O}\right)$ obtained from previous XANES experiments for $\mathrm{La}_{1.94} \mathrm{Sr}_{0.06} \mathrm{CuO}_{4}$ (open circles) [14] and from previous NCFS experiments for $\mathrm{La}_{1.96-x} \mathrm{Ho}_{0.04} \mathrm{Sr}_{x} \mathrm{CuO}_{4}$ $[x=0.15$ (open triangles) [18]; $x=0.11,0.15$ (open squares) [19]] are also displayed. (b) Doping dependence of the isotope effect exponent $\alpha_{T_{c}}$ (solid diamonds) and $\alpha_{T^{*}}$ (solid circles) for $\mathrm{La}_{2-x} \mathrm{Sr}_{x} \mathrm{CuO}_{4}$ as determined in this work, together with published values for $\alpha_{T_{c}}$ (open symbols) [25] and values for $\alpha_{T^{*}}$ extracted from the previous XANES and NCFS results shown in (a): XANES (open circle) [14], NCFS (open triangle) [18], and NCFS (open squares) [19]. The dashed lines are a guide to the eye.

techniques XANES and NCFS yield consistent values for $T^{*}$. Note that for overdoped $\mathrm{La}_{1.96-x} \mathrm{Ho}_{0.04} \mathrm{Sr}_{x} \mathrm{CuO}_{4}(x=0.20$ and $x=0.25$ ), NCFS experiments [19] revealed in contrast to other experiments an unexpected upward shift of $T^{*}$ with an appreciably negative OIE on $T^{*}$ which, however, is not discussed here. 
TABLE I. Summary of the values of the pseudogap temperatures $T^{*}\left({ }^{16} \mathrm{O}\right), T^{*}\left({ }^{18} \mathrm{O}\right)$, and the OIE exponent $\alpha_{T^{*}}$ for $\mathrm{La}_{2-x} \mathrm{Sr}_{x} \mathrm{CuO}_{4}$ $(0.06 \leqslant x \leqslant 0.15)$.

\begin{tabular}{lccc}
\hline \hline Doping $x$ & $T^{*}\left({ }^{16} \mathrm{O}\right)(\mathrm{K})$ & $T^{*}\left({ }^{18} \mathrm{O}\right)(\mathrm{K})$ & $\alpha_{T^{*}}$ \\
\hline 0.06 & $126(10)$ & $187(18)$ & $-4.3(1.1)$ \\
0.09 & $102(12)$ & $135(12)$ & $-2.9(9)$ \\
0.12 & $64(9)$ & $80(10)$ & $-2.2(1.3)$ \\
0.15 & $49(8)$ & $52(8)$ & $-0.6(1.3)$ \\
\hline \hline
\end{tabular}

The corresponding OIE exponent $\alpha_{T^{*}}$ is evaluated analogously to the one on $T_{c}$. It is negative and unusually large with $\alpha_{T^{*}}=-4.4(1.1)$ for the lowest doping $x=0.06$. The magnitude of $\alpha_{T^{*}}$ is decreasing with increasing $x$, reaching a still substantial value of $\alpha_{T^{*}}=-0.6(1.3)$ at optimal doping $x=0.15$ (see Table I). The doping dependencies of the OIE exponents $\alpha_{T^{*}}$ and $\alpha_{T_{c}}$ of $\mathrm{La}_{2-x} \mathrm{Sr}_{x} \mathrm{CuO}_{4}(0.06 \leqslant x \leqslant 0.15)$ are displayed in Fig. 4(b) (solid symbols), together with previous results (open symbols: $\alpha_{T^{*}}[14,18,19], \alpha_{T_{c}}[25]$ ). which are confirmed here.

\section{DISCUSSION}

It is evident from Fig. 4(a) that in the underdoped regime $(0.5 \leqslant x \leqslant 0.15)$ the pseudogap temperatures $T^{*}\left({ }^{16} \mathrm{O}\right)$ and $T^{*}\left({ }^{18} \mathrm{O}\right)$ exhibit almost linear doping dependencies which can be described by the expression

$$
T^{*}\left({ }^{\gamma} \mathrm{O}\right)=\sigma\left({ }^{\gamma} \mathrm{O}\right)\left[x-x_{c}\left({ }^{\gamma} \mathrm{O}\right)\right], \quad \gamma=16,18 .
$$

Here, $\sigma\left({ }^{\gamma} \mathrm{O}\right)$ and $x_{c}\left({ }^{\gamma} \mathrm{O}\right)$ are constants. The present $T^{*}$ data [Fig. 4(a) and Table I] follow Eq. (2), yielding $\sigma\left({ }^{16} \mathrm{O}\right)=-880(100) \mathrm{K}, x_{c}\left({ }^{16} \mathrm{O}\right)=0.201(4)$ and $\sigma\left({ }^{18} \mathrm{O}\right)=$ $-1440(170) \mathrm{K}, x_{c}\left({ }^{18} \mathrm{O}\right)=0.182(3)$. It is interesting to note that both quantities $\sigma$ and $x_{c}$ exhibit a substantial OIE. The physical meaning and the location of the $T^{*}$ line in the phase diagram (temperature versus hole doping) of cuprates have been extensively discussed in the literature and are still heavily debated (see, e.g., Refs. [26-33]). A linear doping dependence of $T^{*}$ has been previously reported for various cuprate systems, and the extrapolated generic doping concentration $x_{c} \simeq 0.19-0.20$ has been discussed in terms of a possible quantum critical point underneath the superconducting dome (see, e.g., Refs. [26-28,31,32]). Note that $x_{c}$ decreases from 0.201(4) to 0.182(3) upon replacing ${ }^{16} \mathrm{O}$ by ${ }^{18} \mathrm{O}$, implying a large positive OIE of about $10 \%$, corresponding to an OIE exponent of $\alpha_{x_{c}}=0.84$ (22) [see Fig. 4(a)]. At present, a theoretical interpretation of this remarkable OIE is not available.

The OIE on $T_{c}$ has been consistently explained earlier using a polaronic approach [8,10,34-37], and within multigap multicondensate superconductivity at the BEC-BCS crossover near a Lifshitz transition in multicomponent electronic systems [38,39]. It is worth mentioning that in an early work, Kresin and Wolf [40] already proposed to explain the OIE on $T_{c}$ and on the penetration depth, considering a strong $c$-axis electron-lattice coupling. More recently, this model was extended by Weyeneth and Müller [10,37] to include a polaronic coupling also to the planes. They succeeded in quantitatively reproducing the experimental data, thus demonstrating the importance of polaron formation for superconductivity in the cuprates.

The basic ingredients of polaron formation are that strong electron-lattice interactions lead to polaron formation, namely, quasiparticles, which renormalize the electronic as well as the lattice degrees of freedom. The squeezing of the electronic hopping integrals has been identified as the origin of the OIE on $T_{c}$ [8]. Here, we argue that the OIE on $T^{*}$ is caused by polaron formation related to an incipient finite momentum lattice instability which manifests itself as a divergence in $\mathrm{Cu}-\mathrm{O}$ mean-square displacement at $T^{*}$ as has been observed in Refs. [41,42].

The primary semiclassical response of the lattice to polaron formation is a rigid harmonic oscillator shift, i.e., the phonon creation and annihilation operators $b^{+}$and $b$ transform to

$$
\tilde{b}_{q}=b_{q}+\sum_{q} \gamma_{i}(q) c_{i}^{+} c_{i}, \quad \tilde{b}_{q}^{+}=b_{q}^{+}+\sum_{q} \gamma_{i}(q) c_{i}^{+} c_{i},
$$

with $c_{i}^{+}$and $c_{i}$ being site $i$ dependent electron creation and annihilation operators and $\gamma_{i}(q)$ the momentum $q$ dependent coupling constants. As a consequence of this coupling, the lattice mode frequencies adopt a temperature dependence $[34,35,43]$,

$$
\tilde{\omega}_{q, j}^{2}=\tilde{\omega}_{0 q, j}^{2}-\frac{\gamma_{q, j}^{2}}{N\left(E_{F}\right)} \sum_{k} \frac{1}{\varepsilon(k)} \tanh \frac{\varepsilon(k)}{k_{B} T},
$$

where $N\left(E_{F}\right)$ is the density of states at the Fermi level, $\varepsilon(k)$ the Fourier transform of the site representation of the electronic bands, and $\tilde{\omega}_{0 q, j}$ the bare branch $j$ and momentum $q$ dependent lattice mode frequency. The electronic dispersions are explicitly given by a local density approximation (LDA) derived form, namely,

$$
\begin{aligned}
\varepsilon(k)= & -2 t_{1}\left[\cos \left(k_{x} a\right)+\cos \left(k_{y} b\right)\right]+4 t_{2} \cos \left(k_{x} a\right) \cos \left(k_{y} b\right) \\
& +2 t_{3}\left[\cos \left(2 k_{x} a\right)+\cos \left(2 k_{y} b\right)\right] \\
& \mp t_{4}\left[\cos \left(k_{x} a\right)-\cos \left(k_{y} b\right)\right]^{2} / 4-\mu,
\end{aligned}
$$

where $t_{1}, t_{2}, t_{3}$, and $t_{4}$ are first, second, third, and interlayer hopping integrals, respectively, and $\mu$ is the chemical potential which controls the band filling and is set equal to zero. Here, $a$ and $b$ are the lattice constants in the $\mathrm{CuO}_{2}$ planes. It is important to note that also the electronic degrees of freedom are renormalized, which leads to an exponential band narrowing for all hopping elements according to the replacement $t \rightarrow$ $t \exp \left[-\gamma^{2} \operatorname{coth}\left(\hbar \omega / 2 k_{B} T\right)\right]$ and a rigid level shift.

From Eq. (4) finite momentum mode softening can occur if the normal mode unrenormalized frequency $\tilde{\omega}_{0 q, j}$ is reduced by the electronic energy, corresponding to $\tilde{\omega}_{q, j}^{2} \rightarrow 0$. Obviously, this situation is controlled by the dependence of the coupling strength which we use as a variable to calculate the mode freezing temperature where $\tilde{\omega}_{q, j}^{2}=0$. Since the electronic energies due to their coupling to the lattice degrees of freedom and the unrenormalized mode energies are both isotope dependent, the freezing temperature is isotope dependent as well. This dependence is huge and sign reversed [43] as compared to the isotope effect on the harmonic lattice mode frequencies and mirrors the isotope effect on the pseudogap onset temperature $T^{*}$, which is identified as the freezing temperature. $T^{*}$ signals the onset of a dynamically 
modulated, patterned structure (composed of local coherent polarons), where the superstructure modulation is defined by the $q$ value, where $\tilde{\omega}_{q, j}^{2}=0$ and $T^{*}$ is defined through the implicit relation

$$
\tilde{\omega}_{0 q, j}^{2}=\frac{\gamma_{q, j}^{2}}{N\left(E_{F}\right)} \sum_{k} \frac{1}{\varepsilon(k)} \tanh \frac{\varepsilon(k)}{k_{B} T^{*}} .
$$

The $\mathrm{Cu}-\mathrm{O}$ mean-square displacement, which is momentum $q$ and branch $j$ dependent, can be expressed as

$$
\sigma^{2}(T)=\hbar /\left(M \tilde{\omega}_{q, j}\right) \operatorname{coth}\left(\hbar \tilde{\omega}_{q, j} / 2 k_{B} T\right),
$$

where $M$ is the oxygen ion mass [43]. As has been shown in Ref. [43], the OIE on $T_{c}$ is caused by the renormalization of the band energies where specifically the second-nearestneighbor hopping integral yields the correct OIE. This suggests a substantial involvement of a $Q_{2}$-type Jahn-Teller mode in the formation of the pseudogap phase which has already been deduced from the early data for the OIE on $T^{*}[15,17,18]$. This is further supported by the observation of a ${ }^{63} \mathrm{Cu} /{ }^{65} \mathrm{Cu}$ isotope effect which is present only in $\mathrm{HoBa}_{2} \mathrm{Cu}_{4} \mathrm{O}_{8}$ [17], but absent in $\mathrm{La}_{1.81} \mathrm{Ho}_{0.04} \mathrm{Sr}_{0.15} \mathrm{CuO}_{4}$ [18], since in the former an umbrella type mode including $\mathrm{Cu}$ ion displacement is active, which is absent in $\mathrm{La}_{2-x} \mathrm{Sr}_{x} \mathrm{CuO}_{4}$. Alternatively, the OIE on $T^{*}$ (but not on $T_{c}$ ) has been explained within a purely electronic model, where lattice effects were built in at specific momentum values [44].

In the above we have not addressed the question why the ${ }^{63} \mathrm{Cu}$ NQR relaxation study [13], opposite to XANES [14] and NCFS [15-19], detects only a small OIE on $T^{*}$ with reversed sign as compared to the present results. First, the $T_{1}$ spinlattice relaxation measurements performed by NQR probe both magnetic and charge fluctuations. However, magnetic fluctuations dominate in the temperature region investigated. Second, the probing time scales of both techniques are quite different, namely, NQR is slow and works in the $\mathrm{kHz}$ to $\mathrm{MHz}$ range, whereas XANES is fast $\left(10^{-15} \mathrm{~s}\right)$ and insensitive to magnetism. This means that NQR and XANES do not probe the same physical quantities, and thus the results obtained for the pseudogap are not directly comparable to each other.

\section{CONCLUSION}

In conclusion, we have performed a systematic investigation of the oxygen isotope effect on the pseudogap temperature $T^{*}$ by using XANES and thereby demonstrated its doping dependent evolution. The isotope effect is always sign reversed and large and increases with decreasing doping. The correct trends of the isotope effect are obtained within a polaronic model, where the renormalization of lattice and electronic degrees of freedom are taken into account to calculate the isotope effect on $T^{*}$. The present results support the original concept, leading to the discovery of high-temperature superconductivity in the cuprates [1] that a $Q_{2}$-type Jahn-Teller mode is at work in the formation of the pseudogap phase, as has been concluded previously in explaining the OIE on $T_{c}$ $[36,37]$. In addition, the data substantiate the notion of the vibronic character of the ground state.

\section{ACKNOWLEDGMENTS}

It is a pleasure to acknowledge the year long support from K. A. Müller, who had already suggested performing this work several years ago. In addition, numerous encouraging discussions with him are gratefully recognized. We also thank B. Joseph, M. Mali, C. Marini, and J. Roos for helpful discussions. Financial support by the Swiss National Science Foundation is gratefully acknowledged. We thank Diamond Light Source for access to beamline B18 (Proposal No. SP9960) that contributed to the results presented here.
[1] J. G. Bednorz and K. A. Müller, Z. Phys. B 64, 189 (1986).

[2] For a review, see, e.g., T. Moriya and K. Ueda, Adv. Phys. 49, 555 (2000), and references therein.

[3] J. Bardeen, L. N. Cooper, and J. R. Schrieffer, Phys. Rev. 108, 1175 (1957).

[4] J. P. Franck, in Physical Properties of High Temperature Superconductors $I V$, edited D. M. Ginsberg (World Scientific, Singapore, 1994), p. 189.

[5] G. M. Zhao, H. Keller, and K. Conder, J. Phys.: Condens. Matter 13, R569 (2001).

[6] H. Keller, in Superconductivity in Complex Systems, edited by K. A. Müller and A. Bussmann-Holder, Structure and Bonding Vol. 114 (Springer, Berlin, 2005), pp. 114-143.

[7] T. Schneider, Phys. Status Solidi B 242, 58 (2005).

[8] H. Keller, A. Bussmann-Holder, and K. A. Müller, Mater. Today 11, 38 (2008).

[9] R. Khasanov, A. Shengelaya, D. Di Castro, E. Morenzoni, A. Maisuradze, I. M. Savić, K. Conder, E. Pomjakushina, A. Bussmann-Holder, and H. Keller, Phys. Rev. Lett. 101, 077001 (2008)

[10] S. Weyeneth and K. A Müller, J. Supercond. Novel Magn. 24, 1235 (2011).
[11] Z. Guguchia, R. Khasanov, M. Bendele, E. Pomjakushina, K. Conder, A. Shengelaya, and H. Keller, Phys. Rev. Lett. 113, 057002 (2014).

[12] G. V. M. Williams, J. L. Tallon, J. W. Quilty, H. J. Trodahl, and N. E. Flower, Phys. Rev. Lett. 80, 377 (1998).

[13] F. Raffa, T. Ohno, M. Mali, J. Roos, D. Brinkmann, K. Conder, and M. Eremin, Phys. Rev. Lett. 81, 5912 (1998).

[14] A. Lanzara, G.-m. Zhao, N. L. Saini, A. Bianconi, K. Conder, H. Keller, and K. A. Müller, J. Phys.: Condens. Matter 11, L541 (1999).

[15] D. Rubio Temprano, J. Mesot, S. Janssen, K. Conder, A. Furrer, H. Mutka, and K. A. Müller, Phys. Rev. Lett. 84, 1990 (2000).

[16] D. Rubio Temprano, A. Furrer, K. Conder, and H. Mutka, Physica B 276-278, 762 (2000).

[17] D. Rubio Temprano, J. Mesot, S. Janssen, K. Conder, A. Furrer, A. Sokolov, V. Trounov, S. M. Kazakov, J. Karpinski, and K. A. Müller, Eur. Phys. J. B 19, 5 (2001).

[18] D. Rubio Temprano, K. Conder, A. Furrer, H. Mutka, V. Trounov, and K. A. Müller, Phys. Rev. B 66, 184506 (2002).

[19] P. S. Häfliger, A. Podlesnyak, K. Conder, E. Pomjakushina, and A. Furrer, Phys. Rev. B 74, 184520 (2006), and references therein. 
[20] For a review, see, e.g., P. A. Lee, N. Nagaosa, and X.-G. Wen, Rev. Mod. Phys. 78, 17 (2006), and references therein.

[21] For a recent review, see, e.g., P. A. Lee, Phys. Rev. X 4, 031017 (2014).

[22] C. Li, M. Pompa, A. Congiu-Castellano, S. Della Longa, and A. Bianconi, Physica C 175, 369 (1991).

[23] A. J. Dent, G. Cibin, S. Ramos, S. A. Parry, D. Gianolio, A. D. Smith, S. M. Scott, L. Varandas, S. Patel, M. R. Pearson, L. Hudson, N. A. Krumpa, A. S. Marsch, and P. E. Robbins, J. Phys.: Conf. Ser. 430, 012023 (2013).

[24] R. Comin and A. Damascelli, Annu. Rev. Condens. Matter Phys. 7, 369 (2016), and references therein.

[25] M. K. Crawford, W. E. Farneth, E. M. McCarron, R. L. Harlow, and A. H. Moudden, Science 250, 1390 (1990); G. M. Zhao, K. Conder, H. Keller, and K. A. Müller, J. Phys.: Condens. Matter 10, 9055 (1998); J. P. Franck, S. Harker, and J. H. Brewer, Phys. Rev. Lett. 71, 283 (1993); J. Hofer, K. Conder, T. Sasagawa, G.-m. Zhao, M. Willemin, H. Keller, and K. Kishio, ibid. 84, 4192 (2000); B. Batlogg, G. Kourouklis, W. Weber, R. J. Cava, A. Jayaraman, A. E. White, K. T. Short, L. W. Rupp, and E. A. Rietman, ibid. 59, 912 (1987).

[26] C. M. Varma, Phys. Rev. B 55, 14554 (1997).

[27] S. Sachdev, Science 288, 475 (2000).

[28] J. L. Tallon and J. W. Loram, Physica C 349, 53 (2001).

[29] M. R. Norman, D. Pines, and C. Kallin, Adv. Phys. 54, 715 (2005).

[30] S. Hüfner, M. A. Hossain, A. Damascelli, and G. A. Sawatzky, Rep. Prog. Phys. 71, 062501 (2008).

[31] Y. Li, V. Balédent, N. Barisić, Y. Cho, B. Fauqué, Y. Sidis, G. $\mathrm{Yu}, \mathrm{X}$. Zhao, P. Bourges, and M. Greven, Nature (London) $\mathbf{4 5 5}$, 372 (2008).
[32] A. Shekhter, B. J. Ramshaw, R. Liang, W. N. Hardy, D. A. Bonn, F. F. Balakirev, R. D. McDonald, J. B. Betts, S. C. Riggs, and A. Migliori, Nature (London) 498, 75 (2013).

[33] B. Keimer, S. A. Kivelson, M. R. Norman, S. Uchida, and J. Zaanen, Nature (London) 518, 179 (2015).

[34] A. Bussmann-Holder and H. Keller, Eur. Phys. J. B 44, 487 (2005).

[35] A. Bussmann-Holder and H. Keller, in Polarons in Advanced Materials, edited by A. S. Alexandrov, Springer Series in Materials Science Vol. 103 (Springer, Berlin, 2007), pp. 599621.

[36] K. A. Müller, J. Phys.: Condens. Matter 19, 251002 (2007).

[37] K. A. Müller, J. Supercond. Novel Magn. 27, 2163 (2014).

[38] A. Perali, D. Innocenti, A. Valletta, and A. Bianconi, Supercond. Sci. Technol. 25, 124002 (2012).

[39] A. Bianconi, A. Valletta, A. Perali, and N. L. Saini, Solid State Commun. 102, 369 (1997).

[40] V. Z. Kresin and S. A. Wolf, Phys. Rev. B 49, 3652 (1994); A. Bill, V. Z. Kresin, and S. A. Wolf, ibid. 57, 10814 (1998).

[41] A. Bianconi, N. L. Saini, A. Lanzara, M. Missori, T. Rossetti, H. Oyanagi, H. Yamaguchi, K. Oka, and T. Ito, Phys. Rev. Lett. 76, 3412 (1996).

[42] H. Oyanagi, C. Zhang, A. Tsukada, and M. Naito, J. Supercond. Novel Magn. 22, 165 (2009).

[43] H. Keller and A. Bussmann-Holder, Adv. Condens. Matter Phys. 2010, 393526 (2010); A. Bussmann-Holder, H. Keller, J. Mustre de Leon, A. Simon, A. R. Bishop, and K. A. Müller, J. Supercond. Novel Magn. 23, 295 (2010).

[44] S. Andergassen, S. Caprara, C. Di Castro, and M. Grilli, Phys. Rev. Lett. 87, 056401 (2001). 